\title{
OPTIMAL PATH PLANNING PROGRAM FOR AUTONOMOUS SPEED SPRAYER IN ORCHARD USING ORDER-PICKING ALGORITHM
}

\author{
T. S. Park ${ }^{1}$, S. J. Park ${ }^{2}$, K. Y. Hwang ${ }^{3}$, S. I. Cho ${ }^{*}$ \\ ${ }^{1}$ Department of Biosystems \& Biomaterials Science and Engineering, Research Institute for \\ Agriculture and Life Sciences, Seoul National University, Seoul, 151-921, Rep. Korea. \\ ${ }^{2}$ Interdisciplinary Program for Bioengineering, Seoul National University, Seoul, 151-921, \\ Rep. Korea. \\ 3 Department of Biosystems \& Biomaterials Science and Engineering, Seoul National \\ University, Seoul, 151-921, Rep. Korea. \\ * Corresponding author: Department of Biosystems \& Biomaterials Science and Engineering, \\ Seoul National University, 599 Gwanangno, Gwanak-gu, 151-921, Rep. Korea.; phone: \\ +82-2-880-4606; fax: +82-2-873-2049; e-mail: sicho@snu.ac.kr.
}

Abstract: This study was conducted to develop a software program which computes optimal path for autonomous navigation in orchard, especially for speed sprayer. Possibilities of autonomous navigation in orchard were shown by other researches which have minimized distance error between planned path and performed path. But, research of planning an optimal path for speed sprayer in orchard is hardly founded. In this study, a digital map and a database for orchard which contains GPS coordinate information (coordinates of trees and boundary of orchard) and entity information (heights and widths of trees, radius of main stem of trees, disease of trees) was designed. An orderpicking algorithm which has been used for management of warehouse was used to calculate optimum path based on the digital map. Database for digital map was created by using Microsoft Access and graphic interface for database was made by using Microsoft Visual $\mathrm{C}++6.0$. It was possible to search and display information about boundary of an orchard, locations of trees, daily plan for scattering chemicals and plan optimal path on different orchard based on digital map, on each circumstance (starting speed sprayer in different location, scattering chemicals for only selected trees).

In efficiency, developed path from this program was compared to conventional path by calculating travel distance on several occasions. Optimum path for rectangular-shaped orchards traveling whole trees showed the same working path as conventional method. But, for irregular-shaped orchards, the program developed in 5\% shorter path than conventional method.

Please use the following format when citing this chapter:

Park, T.S., Park, S.J., Hwang, K.Y. and Cho, S.I., 2009, in IFIP International Federation for Information Processing, Volume 293, Computer and Computing Technologies in Agriculture II, Volume 1, eds. D. Li, Z. Chunjiang, (Boston: Springer), pp. 9-18. 
When optimum path was created to travel randomly selected trees, the program developed in $11.5 \%$ shorter path. The program always developed shorter path on any shape of orchard. Order-picking algorithm based optimal path developing program in orchard showed efficiency of traveling distance and expected to present path which lead working time and cost efficiently in orchard.

\section{INTRODUCTION}

Research has been progressed for unmanned autonomous driving for agricultural machine. Possibility of unmanned autonomous was confirmed from identifying basic information of field (Blackmore, 1993), identifying objects in real-time (Toda, 1993). Research of unmanned autonomous driving could be divided with agricultural chemical spraying algorithms areas such as artificial intelligent controller for backward drive on trailer attached tractor (Cho, 1995) and actual system development with variety sensors such as image processing, ultrasound sensors, terrestrials magnetism sensors and Global Positioning System (Ki, 1996).

An agricultural chemical spraying for fruit orchards is essential tasks that prevent the loss of production from 30 to 35 percent (Cho, 1996). Despite of farmer wearing protective device, pesticide poisoning incident and damages appears from pesticides penetrative on skin. Therefore, unmanned speed sprayer on orchard had to be researched and system developed. Remote control and wireless communications within a grove induced by the cable of an unmanned autonomous driving speed sprayer (Jang, 1995), application with differential global positioning system (DGPS), ultrasonic sensor and fuzzy control (Lee, 1998), e-map based DGPS fuzzy control (Lee, 1999) are those cases.

One of important element is decision making for autonomous driving path. Efficiency gets improved by using electronic maps. In case of orchards, most of driving direction for machines is keep circling between trees. So efficiency of travel distance was not contributed.

When specific points of trees have to be cared in orchard, efficiency could be necessary. In case of partial operation needed on specific trees, optimum path should be decided for unmanned autonomous speed sprayer. Therefore, development of the optimum path decision making program is necessary. On this study, solution of optimum path for orchard by using order-picking algorithm was developed (Cho, 2002). Order-picking algorithm was first started on finding goods on warehouse to optimize movement distance.

The concrete purposes of this study are as follows.

1) Making electronic map which has location and breeding information of trees in orchard by using DGPS. 
2) Developing software program for shortest path on speed sprayer's using order-picking algorithms based on information of electronic map.

3) Evaluation of created work path on custom driving path.

4) Evaluation of created work path on case of specific trees has to be treated Stress-strain experiment

\section{MATERIALS AND METHODS}

\subsection{Unmanned autonomous speed sprayer}

Possibility of trailer attached tractor driving backward (Cho \& Ki, 1995) has shown and it means it is much easy for speed sprayer controlled to drive backward. Autonomous speed sprayer was considered that driving backward is possible because for order-picking algorithm to be applied. This study was conducted base on hypothesis of autonomous speed sprayer with DGPS and electronic map and also can be controlled to be move backward.

\subsection{The configuration of electronic map}

\subsubsection{The configuration of database}

Database was constructed with location information such as boundary of orchard, positions of trees; breeding information such as heights, width, radius of trunk, disease appeared; pesticides treated dates, trees and which trees has to be treated. Those were developed with Microsoft Access program. Table 1 indicates the database table and field.

Table 1 Database table used for attributes

\begin{tabular}{ll}
\hline Table name & Table field \\
\hline \multirow{2}{*}{ FIELD_NAME } & Name of the field \\
& Distance between trees \\
BOUNDARY_MAP & Distance between columns of trees \\
Coordinates of boundary points & Date of scattering \\
WORK_DATE & ID of the field to be scattered \\
TREE & Coordinates \\
SPRAY & Entity of trees \\
& ID of trees to be scattered \\
DISEASE_NAME & Name of disease \\
\end{tabular}




\subsubsection{Boundaries of the orchard, location of orchards and making route points map}

Boundary maps are connected in the database stored the each point of BOUNDARY_MAP table and it to pull up the map in a straight line. When to use it, it can be possible that operation speed sprayer within operation boundaries the move this judgment, and also to determine where you can.

Information about the location of orchards is marked on the boundary map using the information in their fields in TREE table of database and is indicated in two circles that are the thickness of trunk and distance between trees.

The map of middle route points is indicated in mark that is set in passing point of speed sprayer considering speed sprayer of rotation and condition of the operation path.

The data in the database is mapped from using Microsoft Visual $\mathrm{C}++6.0$ and figure 1 is three types of implementing a virtual map of the orchard. Numerical values in the figures are shown the latitude and longitude, as GPS location data

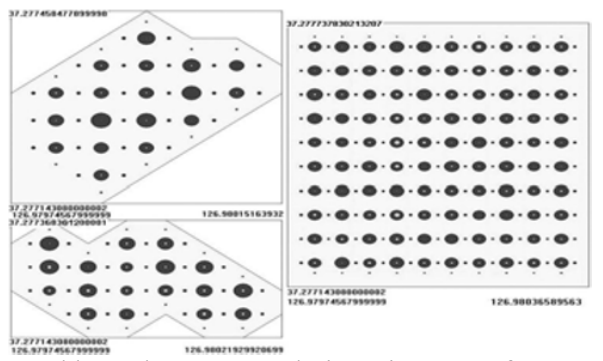

Fig. 1 Programmed boundary, tree and via-point map of 3 types of field map.

(Field type 1: left top, 2: left bottom, 3: right)

\subsection{Making operation path as using electronic map}

\subsubsection{Assumption of agricultural chemical spraying}

It assumes that the speed sprayer passes all the passage of orchards and spray twice to minimize the time of agricultural chemical spraying and equal agricultural chemical spraying operation passage(Figure 2.b). And It assumes that only with the vertical direction of the driving speed sprayer can't rotate 180-degree in the middle column of orchards considering the reality of the orchard. Because It assumes to spray twice the middle of orchards in same column sets point of agricultural chemical spraying. If orchards do not have the same column in the orchards, the half of distance 
between trees on orientating vertical direction of speed sprayer sets point of agricultural chemical spraying.

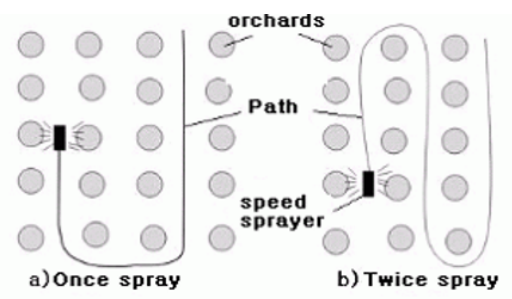

Fig. 2 Method of spraying chemicals for speed sprayer on orchard.

\subsubsection{Order-picking algorithm using dynamic programming}

Dynamic programming that is multi-level decision-making across the dynamic programming for the optimization is method that solve problem of cascading decision on the issue, through the decomposition or split while reducing the size of the problem. Order-picking is algorithms for efficient performing process that the machine items of orders from customers. It assumes that the position of the goods in warehouse is orchards that need agricultural chemical spraying.

The following description of the physical location of my orchards and orchards can be expressed as in Figure 3 represents the following explanation. The physical location of the orchards in orchard is the following explanation and figure 3 shows the following explanation. Moving path is from the first column of orchards on left including orchards 1 to first column of orchards on right including orchards $\mathrm{r}$. If $T_{i \text { is partial path for agricultural }}$ chemical spraying, it can be divided into two different routes.

$a_{i}$ : the ending point of pathway $i$

$b_{i}$ : the starting point of pathway $i$

$T_{i}^{a}$ : the entry on the pathway $i$

$T_{i}^{b}$ : the entry under the pathway $i$

The method of moving pathway $i-1$ to $i$ is the following two ways.

$t_{a}$ : In the case of moving on the column of orchards

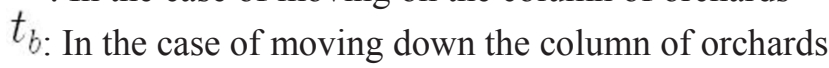

The method of agricultural chemical spraying orchards in pathway $i$ is the following four ways.

$t_{1}$ : In the case of moving the pathway

$t_{2}$ : In the case of no entry the pathway

$t_{3}$ : In the case of the entry under the block then exiting out again 
$t_{4}$ : In the case of the entry on the block then exiting out again

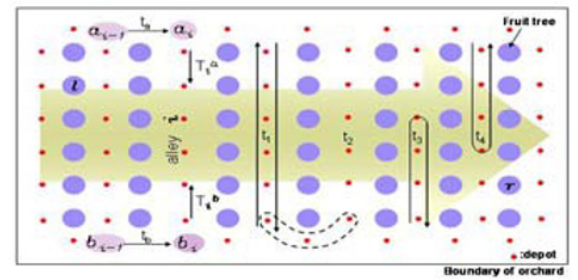

Fig. 3 Possible traveling path of speed sprayer in orchard.

$\mathrm{A}^{t_{2}}$ is in the case of no agricultural chemical spraying orchards in pathway. Partial path $T_{i}$ is above-mentioned $t_{w}(w=1,2,3,4, \mathrm{a}, \mathrm{b})$ and $T_{i}+t_{w}$. The time that speed sprayer pass path $T_{i}+t_{w}$ is $f\left(T_{i}+t_{w}\right)$.

Procedures are as follows for the creation of order-picking.

Step 1 : The dependant path that include agricultural chemical spraying orchards $l$ (column of orchards closed with boundaries at the start of agricultural chemical spraying)is the following two ways.

$T_{l}^{a}$ : From node $a_{l}$ to node ${ }^{a_{l}}$ and step is progressed.

$T_{l}^{a}$ : From node $a_{l}$ to node $b_{l}$ and step is progressed.

$T_{l}^{b}$ : From node $b_{l \text { to node }} a_{l}$ and step is progressed.

$T_{l}^{b}$ : From node $b_{l \text { to node }} b_{l}$ and step is progressed.

Step 2 : If each of the successive path $i$ locates between path $l$ and $r$ and includes agricultural chemical spraying orchards, $T_{i}^{a}$ and $T_{i}^{b}$ are as follows.

$$
\begin{aligned}
& T_{i}^{a}=T_{i-1}^{a}+t_{a}+t_{4} \text { if, } f\left(T_{i-1}^{a}+t_{a}+t_{4}\right)<f\left(T_{i-1}^{b}+t_{b}+t_{1}\right. \\
& T_{i}^{a}=T_{i-1}^{b}+t_{b}+t_{1}, \text { otherwise } \\
& T_{i}^{b}=T_{i-1}^{b}+t_{b}+t_{3} \text { if, } f\left(T_{i-1}^{b}+t_{b}+t_{3}\right)<f\left(T_{i-1}^{a}+t_{a}+t_{1}\right. \\
& T_{i}^{b}=T_{i-1}^{a}+t_{a}+t_{1}, \text { otherwise }
\end{aligned}
$$

If path $i$ doesn't include agricultural chemical spraying orchards and are as follows.

$$
\begin{aligned}
& T_{i}^{a}=T_{i-1}^{a}+t_{a} \\
& T_{i}^{b}=T_{i-1}^{b}+t_{b}
\end{aligned}
$$

Step 3 : Final path(column of orchards closed with boundaries at the end of agricultural chemical spraying) is as follows.

$T_{r}^{a}=T_{r-1}^{a}+t_{a}+t_{4}$ if, $f\left(T_{r-1}^{a}+t_{a}+t_{4}\right)<f\left(T_{r-1}^{b}+t_{b}+t_{1}\right.$

$T_{r}^{a}=T_{r-1}^{b}+t_{b}+t_{1}$, otherwise

$T_{r}^{b}=T_{r-1}^{b}+t_{b}+t_{3}$ if, $f\left(T_{r-1}^{b}+t_{b}+t_{3}\right)<f\left(T_{r-1}^{a}+t_{a}+t_{1}\right.$

$T_{r}^{b}=T_{r-1}^{a}+t_{a}+t_{1}$, otherwise 
Making final operation path is to select the shortest distance $T_{i}^{a}$ and $T_{i}^{b}$ at each step in each step through the process, generated from the above four kinds of path.

\subsection{Making operation path using order-picking algorithm}

The generated path is assumed that starting point of speed sprayer is the outline of orchard. If a number of path that speed sprayer can enter in orchard are many or The farmhouse is located in the middle of orchard so the starting point of operation is not regular, it can't be used directly in creation of path. In order that speed sprayer can pass between trees, 3 passpoints is set up around orchards in consideration of the radius of gyration of first and last orchards on column of orchards(dotted line of figure 3 ). When the starting point of operation is located in orchard(figure 5,7) after orchard is split into two parts, spraying is progressed on one side, then spraying is progressed and contact with former operation on other sides.

\section{RESULT AND INVESTIGATION}

\subsection{Making operation path for all agricultural chemical spraying operations}

Putting three type of orchard, eclectic map is made and changing starting point pf speed sprayer, distance of speed sprayer using operation path of customary method(It can drive only forward) and using developed algorithm are compared and analyzed. Figure 4 shows operation path using customary method and created operation path using order-picking algorithm in irregular field type 2. In each of figures, marked square point is starting point of operation and the color of between two circles is orchards that agricultural chemical spraying is needed.

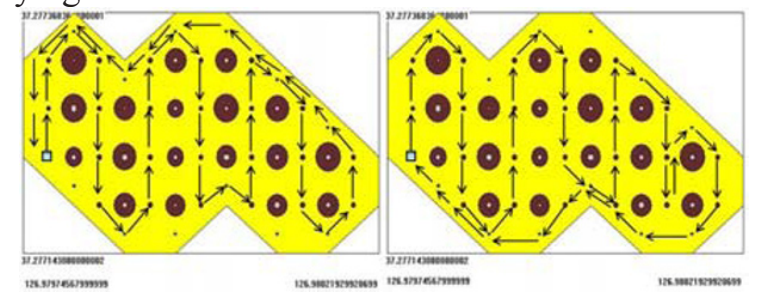

Fig. 4 Comparison of path planned by conventional (left) and order-picking(right) algorithm on field type 2 . 
A total distance is $163.03 \mathrm{~m}$ by using customary method which is consider only straight direction and it is $161.0 \mathrm{~m}$ by using order-picking algorism. The figure 5 shows that variation of direction is related to the prevention of the breeding and extermination depends on the starting point for the matter of field type 1-irregular form. The square in the figure indicates initial position. About three types of field, a moving path of the prevention of the breeding and extermination is calculated by simulating and is described in the table 2 . lc is the operation direction length with customary method, lo is the operation direction with developed algorism. A $\triangle \mathrm{l}$ is divided into a hundred parts of lc and (1c-10). The pathway which is made by rectangular shape field type 3 is formed similar one when using a customary method and there is no difference of moving distance. On the other hand, about a irregular formed field type 1 , it differ from primarily pathway and there is the differences in moving distance.

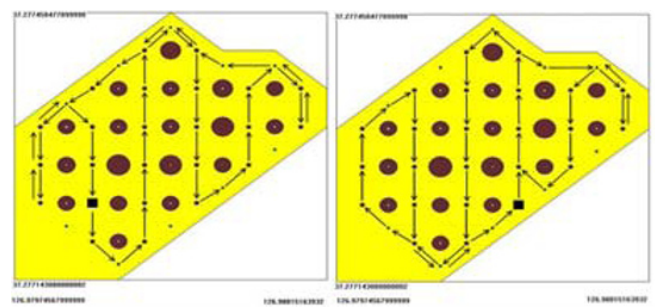

Fig. 5 Path planned by order-picking algorithm by different starting point on field type 1

Table 2 Total path length difference by applying order-picking algorithm on 3 types of field with different starting point

\begin{tabular}{|c|c|c|c|c|c|}
\hline \multirow{2}{*}{ Field type } & \multicolumn{2}{|c|}{ Coordinates of starting points } & \multicolumn{2}{|c|}{ Length of the path } & \multirow{2}{*}{$(\%)$} \\
\hline & Latitude & Longitude & & & \\
\hline \multirow{4}{*}{1} & 37.2772331943 & 126.979779511 & 167.5 & 167.5 & 0 \\
\hline & 37.2771881368 & 126.979914829 & 171.0 & 159.2 & 6.9 \\
\hline & 37.2772782505 & 126.980050149 & 169.0 & 164.3 & 3.6 \\
\hline & 37.2773233073 & 126.980117808 & 172.0 & 162.9 & 5.3 \\
\hline \multirow{4}{*}{2} & 37.2772331937 & 126.979779511 & 163.0 & 160.9 & 1.3 \\
\hline & 37.2771881368 & 126.979914829 & 188.6 & 170.9 & 9.4 \\
\hline & 37.2771881368 & 126.980117808 & 181.9 & 166.1 & 8.7 \\
\hline & 37.2773233073 & 126.980185468 & 178.8 & 169.3 & 5.3 \\
\hline \multirow{4}{*}{3} & 37.2771971482 & 126.979830254 & 774.3 & 774.3 & 0 \\
\hline & 37.2771971482 & 126.979886637 & 767.25 & 767.25 & 0 \\
\hline & 37.2771971482 & 126.979943024 & 770.78 & 770.78 & 0 \\
\hline & 37.2771971482 & 126.979999404 & 767.25 & 767.25 & 0 \\
\hline
\end{tabular}




\subsection{Making operation path for selective agricultural chemical spraying operations}

It is necessary to make the shortest operating path when worker has to select some orchards. Figure 6(a) shows that only path of normally straight driving when it is needed to do work at selected some orchards in field type 1 and the length of all paths is $135.3 \mathrm{~m}$. Figure $6(\mathrm{~b})$ shows formed path using order-picking algorithm and the length of all formed paths is $124.0 \mathrm{~m}$. Figure 7 shows change of agricultural chemical spraying path with change of speed sprayer starting point about irregular field type 1 .
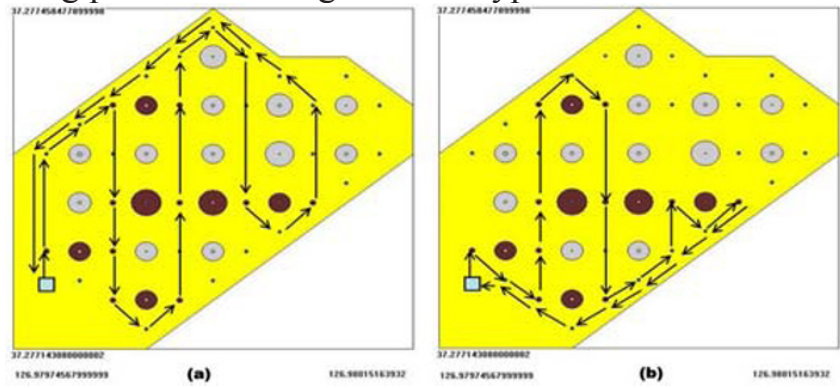

Fig. 6 Path planned for selected trees using (a) conventional algorithm and (b) order-picking algorithm.

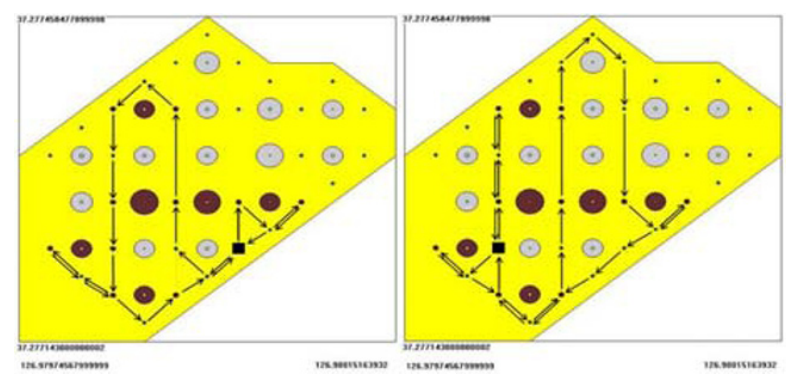

Fig. 7 Path planned for selected trees using order-picking algorithm with different starting point.

Table 3 shows change of length for agricultural chemical spraying path with applied algorithm and change of speed sprayer starting point when the path of agricultural chemical spraying is made with selected some of orchards in irregular field type 1 and rectangular field type 3. created length of pathway is shorter $9.1 \%$ than created length of pathway according to customary method. 


\section{SUMMARY AND CONCLUSION}

In this study, order-picking algorithm using dynamic programming that is used in warehouse is applied in orchard and work path of reversible autonomous speed sprayer is used.

The definite result is followed

1. About all parts of orchard, when agricultural chemical spraying operation is progressed, in rectangular type orchard (field type 3), created path is same with operation path of customary method. But in irregular type orchard, created path shows effect of path reduction at 5.06\%

2. About selected specific parts of orchard, when agricultural chemical spraying operation is progressed, in rectangular type orchard (field type 3), created path is $9.1 \%$ shorter than operation path of customary method (It can drive only forward). Finally, in this study, developed program shows that in case of operation in irregular type orchard is more effective than operation of rectangular type orchard.

\section{REFERENCES}

Blackmore, B. S. and T. Steinhauser. 1993. Intelligent sensing and self-organizing fuzzy logic techniques used in agricultural automation. ASAE paper No. 931048, Summer meeting.

Cho, J. N. 2002. Development of the order picking strategy algorithm for WMS in SCM environment. Masters degree thesis of University of Incheon. (In Korean)

Cho, S. I. and N. H. Ki. 1995. Backward control simulation of tractor-trailer using fuzzy logic and genetic algorithms. Journal of Biosystems Engineering 20(1):87-94. (In Korean)

Cho, S. I. and N. H. Ki. 1996. Autonomous speedsprayer using machine vision and fuzzy logic( I ) -Graphic simulation-. Journal of Biosystems Engineering 21(2):167-174. (In Korean)

Jang, I. J., T. H. Kim and M. D. Cho. 1995. Development of unmanned speedsprayer( I ) Remote Control and induction cable system-. Journal of Biosystems Engineering 20(3):226235. (In Korean)

Ki, N. H., S. I. Cho and C. H. Choi. 1996. Autonomous speedsprayer using machine vision and fuzzy logic( II ) -Real operation-. Journal of Biosystems Engineering 21(2):175-181. (In Korean)

Lee, J. H., S. I. Cho and J. Y. Lee. 1998. Autonomous speedsprayer using DGPS and fuzzy control( II ) -Real operation-. Journal of Biosystems Engineering 23(1):75-82. (In Korean)

Lee, J. -Y. 1999. Autonomous Speedsprayer Using DGPS and GIS. Masters degree thesis of Seoul National University. (In Korean)

Toda, M., O. Kitani, T. Okamoto and T. Tori. 1993. Studies on autonomous vehicles for agricultural robotics. ASAE paper No. 933091, Summer meeting. 